\title{
Third Bose Fugacity Coefficient in One Dimension, As a Function of Asymptotic Quantities
}

\author{
A. Amaya-Tapia ${ }^{1}$, S. Y. Larsen ${ }^{2}$ and M. Lassaut ${ }^{3}$ \\ ${ }^{1}$ Instituto de Ciencias Físicas, Universidad Nacional Autónoma de México AP 48-3, Cuernavaca, \\ Mor. 62251, México. ${ }^{2}$ Department of Physics, Temple University, Philadelphia, PA 19122, USA. \\ ${ }^{3}$ Institut de Physique Nucléaire, IN2P3-CNRS, Université Paris-Sud 11, F-91406 Orsay Cedex, \\ France.
}

\begin{abstract}
In one of the very few exact quantum mechanical calculations of fugacity coefficients, Dodd and Gibbs (J. Math.Phys.,15, 41 (1974)) obtained $b_{2}$ and $b_{3}$ for a one dimensional Bose gas, subject to repulsive delta-function interactions, by direct integration of the wave functions. For $b_{2}$, we have shown (Mol. Phys.,103, 1301 (2005)) that Dodd and Gibbs' result can be obtained from a phase shift formalism, if one also includes the contribution of oscillating terms, usually contributing only in 1 dimension. Now, we develop an exact expression for $b_{3}-b_{3}^{0}$ (where $b_{3}^{0}$ is the free particle fugacity coefficient) in terms of sums and differences of 3-body eigenphase shifts. Further, we show that if we obtain these eigenphase shifts in a distorted-Born approximation, then, to first order, we reproduce the leading low temperature behaviour, obtained from an expansion of the two-fold integral of Dodd and Gibbs. The contributions of the oscillating terms cancel.

The formalism that we propose is not limited to one dimension, but seeks to provide a general method to obtain virial coefficients, fugacity coefficients, in terms of asymptotic quantities. The exact one dimensional results allow us to confirm the validity of our approach in this domain.
\end{abstract}

Keywords Virial Coefficients · Hyperspherical basis · Boson systems

PACS 05.30.Jp $\cdot 05.70 . c e \cdot 34.50 . \tilde{\mathrm{s}}$ 


\section{Introduction}

As demonstrated in Nature 1 the behaviour of quantum models, even in one dimension, and of such gases, remains of current interest! In that paper, the paramount interest is the experimental investigation of a system that is not ergotic and does not equilibrate after thousands of collisions. Still, the description of a quantum mechanical gas, in equilibrium, needs adequate description. So, for example, we note the fairly recent article by Hussien and Yahya [2], on the quantum corrections to the classical fourth virial coefficient for square well potentials.

Our efforts are, instead, directed to the continuing development of a fully quantum mechanical formalism, applicable to the higher virials, and especially useful at low temperatures.

Let us be clear, there is an enormous difficulty in evaluating the required traces in Statistical Mechanics, which involve 3 or more particles. Our adiabatic hyperspherical formalism, uniquely, can 'deal' with 3 or more quantum mechanical particles, whether for Boltzmann or Bose or Fermi statistics, in arbitrary dimensions. This is very important: it can be extended to more particles. The hyperspherical formalism can, in principle, be used to represent an arbitrary number of particles.

Another 'key' element is that we express our results in terms of 'asymptotic' quantities, in our particular case here: 'eigenphase shifts'. This can be done for the 2-body problem, and the essential 'jump' is from two to three and more particles!

We are continuing to extend our formalism, and we use 1-dim to validate our results and to verify that we can carry out our fugacity program. In this paper, we show how we 'deal' with Bose statistics and repulsive forces. Previously, we have limited ourselves to Boltzmann statistics, but developed the present approach which relies on the use of a complete hyperspherical adiabatic basis 3 . 
We recall that, many years ago, Dodd and Gibbs [4] were able to evaluate the second and third virials of identical Bose particles in one dimension, subject to repulsive delta function interactions. To do this, they introduced a complete set of energy eigenfunctions in the traces, and then integrated to obtain answers in terms of single and two-dimensional integrals.

Their original aim, as is clear from their paper and from Gibbs' thesis, was to obtain a formalism in terms of the S-matrix, following the formalism of Dashen, Ma and Bernstein [5], in an effort to obtain the virials in terms of scattering quantities. This was not possible, as the authors determined that the "formal limiting processes are not valid for the singular amplitudes of this system".

An alternative formalism, proposed by Larsen and Mascheroni [6], was a step aimed at ultimately expressing the higher virials in terms of eigenphase shifts and bound state energies, and, if necessary, other on-shell properties, in a hyperspherical harmonic formalism. Under some constraints, one was then able to recover the classical results for the 3rd virial, through a semi-classical approximation [7. Low temperature calculations have been carried out for repulsive potentials in 2 dimensions [8], and a more sophisticated formalism, potentially able to 'handle' correctly the bound states, has been presented for Boltzmann statistics[3]. It involves the use, from the beginning, of a hyperspherical adiabatic basis. Here, we extend that formalism to apply to Bose gases.

To summarize, our aim, in this paper, is to use the excellent results of Dodd and Gibbs to confirm our virial formalism for the third fugacity coefficient, for this particular dimension and interaction, for which we have analytical and numerical results [9, 10, 11. More specifically, we will show that the relevant $b_{3}-b_{3}^{0}$ can be rewritten as an integral, over the energy, of a Boltzmann factor together with the sums and differences of eigenphase shifts, for 3-body wave functions in one dimension. Using, then, a distorted-Born approximation for the eigenphase shifts, we reproduce the leading low temperature behaviour, obtained from an expansion of the two-fold integral of Dodd and Gibbs. 


\section{Statistical Mechanics}

In this section we start with the Grand Partition function in 3 dimensions:

$$
\mathcal{Q}=\sum_{n=0}^{\infty} z^{n} \operatorname{Tr}\left(e^{-\beta H_{n}}\right)=1+z \operatorname{Tr}\left(e^{-\beta T_{1}}\right)+z^{2} \operatorname{Tr}\left(e^{-\beta H_{2}}\right)+z^{3} \operatorname{Tr}\left(e^{-\beta H_{3}}\right)+\cdots
$$

The fugacity $z$ equals $\exp (\mu / \kappa T) ; \beta=1 / \kappa T$, where $\mu, \kappa$ and $T$ are the Gibbs' function per particle, Boltzmann's constant and the temperature, respectively. $H_{n}$ and $T_{n}$ are the n-particle Hamiltonian and kinetic energy operators.

We note that no factorials ( 1 /factorials) appear in this development. This is correct for Bose or Fermi statistics. Also important, is the fact that

$$
\operatorname{Tr}\left(e^{-\beta H_{n}}\right) \rightarrow V^{n} \text { as the volume } V \rightarrow \text { large },
$$

leading to the divergence of the individual traces in the thermodynamic limit. If we take, however, the logarithm of the Grand Partition function $\mathcal{Q}$, we obtain

$$
\begin{aligned}
\ln \mathcal{Q} & =z \operatorname{Tr}\left(e^{-\beta T_{1}}\right) \\
& +z^{2}\left[\operatorname{Tr}\left(e^{-\beta H_{2}}\right)-\frac{1}{2}\left[\operatorname{Tr}\left(e^{-\beta T_{1}}\right)\right]^{2}\right] \\
& +z^{3}\left[\operatorname{Tr}\left(e^{-\beta H_{3}}\right)-\operatorname{Tr}\left(e^{-\beta T_{1}}\right) \operatorname{Tr}\left(e^{-\beta H_{2}}\right)+\frac{1}{3}\left[\operatorname{Tr}\left(e^{-\beta T_{1}}\right)\right]^{3}\right] \\
& +\cdots
\end{aligned}
$$

which, when divided by $\mathrm{V}$, gives coefficients of the powers of $z$, which are independent of the volume, when the latter becomes large. They are the fugacity coefficients $b_{l}$. We can then write for the pressure and the density

$$
\begin{gathered}
p / \kappa T=(1 / V) \ln \mathcal{Q}=\sum_{l} b_{l} z^{l} \\
N / V=\rho=\sum_{l} l b_{l} z^{l},
\end{gathered}
$$

and the fugacity can be eliminated to yield the pressure in terms of the density, in the virial expansion:

$$
\frac{p}{\mathrm{k} T}=\sum_{n=1}^{\infty} B_{n} \rho^{n}
$$

\section{One dimension, three Bose particles}

We follow the procedures outlined in the 'Bogoliubov' lecture 3], developed for Boltzmann particles, and adapt the formalism to Bose Statistics.

From Eq.(2), and changing from the volume $V$ to a length $L$, an expression for the 3rd fugacity coefficient can be written as

$$
b_{3}=\frac{1}{L}\left[\operatorname{Tr}\left(e^{-\beta H_{3}}\right)-\operatorname{Tr}\left(e^{-\beta T_{1}}\right) \operatorname{Tr}\left(e^{-\beta H_{2}}\right)+\frac{1}{3} \operatorname{Tr}\left(e^{-\beta T_{1}}\right)^{3}\right]
$$

Note that each of the 3 terms in the bracket, above, grows as $L^{3}$ asymptotically, for large $L$. Therefore, subtractions must take place, so that $b_{3}$ might become $L$-independent, as is required. If we factor out the contribution of the C.M. (proportional to $L$ ), each term will have a dependence proportional to $L^{2}$.

To help us decrease the $L$ dependence, let us subtract, from each of terms above, the equivalent term without interaction.

Subtracting, therefore, $b_{3}^{0}$ from the $b_{3}$, we obtain:

$$
b_{3}-b_{3}^{0}=\frac{1}{L}\left[\operatorname{Tr}\left(e^{-\beta H_{3}}-e^{-\beta T_{3}}\right)-\operatorname{Tr}\left(e^{-\beta T_{1}}\right) \operatorname{Tr}\left(e^{-\beta H_{2}}-e^{-\beta T_{2}}\right)\right]
$$




\section{Adiabatic Preliminaries}

For the 3 particles of equal masses, in one dimension, we first introduce center of mass and Jacobi coordinates. We define

$$
\eta=\left(\frac{1}{2}\right)^{1 / 2}\left(x_{1}-x_{2}\right) \quad \xi=\left(\frac{2}{3}\right)^{1 / 2}\left(\frac{x_{1}+x_{2}}{2}-x_{3}\right) \quad R=\frac{1}{3}\left(x_{1}+x_{2}+x_{3}\right)
$$

where, of course, the $x_{i}$ give us the locations of the 3 particles. This is a canonical transformation and insures that in the kinetic energy there are no cross terms.

The variables $\xi$ and $\eta$ are involved separately in the Laplacians and we may consider them as acting in different spaces. We introduce a higher dimensional vector $\vec{\rho}=\left(\begin{array}{l}\xi \\ \eta\end{array}\right)$ and express it, here in two dimensions, in a simple polar coordinate system (using the radius $\rho$ and the angle $\vartheta$ ). We write

$$
\eta=\rho \cos \vartheta ; \quad \xi=\rho \sin \vartheta, \quad-\pi<\vartheta \leq \pi ; \quad 0 \leq \rho<\infty .
$$

If we factor a term of $\rho^{1 / 2}$ from the solution of the relative Schrödinger equation, i.e. we let $\psi=\tilde{\phi} / \rho^{1 / 2}$, we are led to:

$$
\left[-\frac{\partial^{2}}{\partial \rho^{2}}+H_{\rho}-k^{2}\right] \tilde{\phi}(k, \vec{\rho})=0
$$

where

$$
H_{\rho}=-\frac{1}{\rho^{2}}\left[\frac{\partial^{2}}{\partial \vartheta^{2}}+\frac{1}{4}\right]+\frac{2 m}{\hbar^{2}} V(\rho, \vartheta)
$$

$m$ is the mass of each particle, $k=\sqrt{2 m E / \hbar^{2}}$ and $E$ is the relative energy in the center of mass.

We now introduce the adiabatic basis, which consists of the eigenfunctions of part of the Hamiltonian: the angular part of the kinetic energy and the potential.

$$
H_{\rho} B_{\ell}(\rho, \vartheta)=\Lambda_{\ell}(\rho) B_{\ell}(\rho, \vartheta),
$$

where $\ell$ enumerates the solutions.

Using this adiabatic basis, we can now rewrite the Schrödinger equation as a system of coupled ordinary differential equations. For a typical solution, we write

$$
\tilde{\phi}(k, \vec{\rho})=\sum_{\ell^{\prime}} B_{\ell^{\prime}}(\rho, \vartheta) \phi_{\ell^{\prime}}(k, \rho)
$$

and obtain the set of coupled equations

$$
\begin{aligned}
\left(\frac{d^{2}}{d \rho^{2}}-\Lambda_{\ell}(\rho)+k^{2}\right) \phi_{\ell}(k, \rho) & +2 \sum_{\ell^{\prime}} C_{\ell}^{\ell^{\prime}}(\rho) \frac{d}{d \rho} \phi_{\ell^{\prime}}(k, \rho) \\
& +\sum_{\ell^{\prime}} D_{\ell}^{\ell^{\prime}}(\rho) \phi_{\ell^{\prime}}(k, \rho)=0
\end{aligned}
$$

where we defined:

$$
\begin{aligned}
C_{\ell}^{\ell^{\prime}}(\rho) & =\int d \vartheta B_{\ell}^{*}(\vartheta, \rho) \frac{\partial}{\partial \rho} B_{\ell^{\prime}}(\vartheta, \rho) \\
D_{\ell}^{\ell^{\prime}}(\rho) & =\int d \vartheta B_{\ell}^{*}(\vartheta, \rho) \frac{\partial^{2}}{\partial \rho^{2}} B_{\ell^{\prime}}(\vartheta, \rho) .
\end{aligned}
$$




\section{Three binary interactions: case (123)}

In our model calculation, the interaction between our particles will be a repulsive delta-function, with 'strength' $g>0$. For 3 particles, we will thus have an interaction potential $V(\rho, \vartheta)$ :

$$
V(\rho, \vartheta)=g[\delta(\sqrt{2} \rho|\cos \vartheta|)+\delta(\sqrt{2} \rho|\cos (\vartheta-2 \pi / 3)|)+\delta(\sqrt{2} \rho|\cos (\vartheta+2 \pi / 3)|)] .
$$

In a set of previous papers [9-15] we, and colleagues, have studied these systems (with $g$ positive and negative), calculated eigenpotentials, adiabatic bases, phase shifts, eigenfunctions and binding energies - where appropriate. We refer to the Appendices for a selection of results, that we need in our present calculations.

The adiabatic eigenfunctions $B_{K}(\rho, \vartheta)$ can be characterized by their symmetry, together with the index $K$, as they reduce to simple normalized surface harmonics when the potential $V(\rho, \vartheta) \rightarrow 0$ and their associated eigenvalues $\Lambda_{K} \rightarrow\left(K^{2}-\frac{1}{4}\right) / \rho^{2}$.

For these surface harmonics, in the completely symmetric 1-dimensional representation $\Gamma_{1}$, we need [12] to set $K=0 \bmod 3$. The harmonics will then be:

$$
\begin{aligned}
& \text { for } K=\text { even, } \quad \frac{1}{\sqrt{\pi}} \cos (K \vartheta) \quad \text { with } \frac{1}{\sqrt{2 \pi}} \text { for } K=0 \\
& \text { for } K=\text { odd, } \quad \frac{1}{\sqrt{\pi}} \sin (K \vartheta)
\end{aligned}
$$

[The completely antisymmetric 1-dimensional representation $\bar{\Gamma}_{1}$ requires, in the above, to interchange the role of the sines and cosines (clearly without the constant term). Finally, in cases where $K \neq 0 \bmod 3$, the sines and cosines form pairs belonging to a 2-dimensional representation $\Gamma_{2}$. ]

The $B_{K}(\rho, \vartheta)$ can then be constructed from the harmonics or obtained as solutions of a differential equation, Eq.(6). The resulting forms are then[14]:

$$
\begin{array}{llll}
\text { for } K=\text { even, } & A_{K}(\rho) \cos \left(q_{K}\left(\vartheta-\frac{m \pi}{3}\right)\right) & \text { where } q_{K} \text { satisfies } & q_{K} \tan \left(\frac{\pi q_{K}}{6}\right)=\frac{\pi \rho c}{6} \\
\text { for } K=\text { odd, } & A_{K}(\rho) \sin \left(q_{K}\left(\vartheta-\frac{m \pi}{3}\right)\right) & \text { where } q_{K} \text { satisfies } & q_{K} \cot \left(\frac{\pi q_{K}}{6}\right)=-\frac{\pi \rho c}{6}
\end{array}
$$

and where $m$ is an integer such that $|\vartheta-m \pi / 3|<\pi / 6$ and $c=\left(2 m / \hbar^{2}\right)(3 g / \pi \sqrt{2})$.

For each value of $\rho$, we need to solve the transcendental equation for $q_{K}$. We can do so numerically, or alternatively seek expansions in $\rho$ or $1 / \rho$, for small or large values of $\rho$, respectively.

$A_{K}(\rho)$ is simply the normalizing factor, such that the $B_{K}(\rho, \vartheta)$ are orthonormal when integrating over the angle, with a choice of integrating over a 'sector', say $-\pi / 6$ to $\pi / 6$, or over a more extended range such as from 0 to $2 \pi$.

\section{Two interacting particles + a spectator: case $(12,3)$}

In this case, which we also require, we use our 3-body formalism to treat three particles, only two of which are interacting, the other one playing the role of a spectator. It is very important that this be done in a way closely paralleling the treatment for the three interacting particles, because we will need to subtract traces, associated with lengths (volumes in 3-dimensions) and they must be evaluated in a similar way in all of the terms of our expressions.

Since our need is the evaluation of traces, we can choose particles 1 and 2 as interacting, particle 3 as the spectator. Letting $\vartheta \rightarrow-\vartheta+\pi$, and therefore letting $\eta$ go to $-\eta$ and $\xi$ go to $\xi$, interchanges the role of particles 1 and 2. The symmetrical combinations for the harmonics (i.e. which treat particles 1 and 2 in a symmetrical fashion) will then be:

$$
\begin{aligned}
& \text { for } K=\text { even, } \quad \frac{1}{\sqrt{\pi}} \cos (K \vartheta) \quad \text { with } \frac{1}{\sqrt{2 \pi}} \text { for } K=0 \\
& \text { for } K=\text { odd, } \quad \frac{1}{\sqrt{\pi}} \sin (K \vartheta)
\end{aligned}
$$

where, of course, there is no longer a restriction of $0 \bmod 3 . K$ now runs over $0,2,4,6 \ldots$ or $1,3,5 \ldots$. 
The $B_{K}^{12,3}(\rho, \vartheta)$ will read:

$$
\begin{aligned}
& \text { for } K=\text { even, } \quad A_{K}^{12,3}(\rho) \cos \left(q_{K}(\vartheta-m \pi)\right) \quad \text { where } q_{K} \text { satisfies } \quad q_{K} \tan \left(\frac{\pi q_{K}}{2}\right)=\frac{\pi \rho c}{6} \\
& \text { for } K=\text { odd, } \quad A_{K}^{12,3}(\rho) \sin \left(q_{K}(\vartheta-m \pi)\right) \quad \text { where } q_{K} \text { satisfies } \quad q_{K} \cot \left(\frac{\pi q_{K}}{2}\right)=-\frac{\pi \rho c}{6}
\end{aligned}
$$

and where $m$ is an integer such that $|\vartheta-m \pi|<\pi / 2$ and, again, $c=\left(2 m / \hbar^{2}\right)(3 g / \pi \sqrt{2})$.

The $A_{K}^{12,3}(\rho)$ are the normalizing factors, such that the $B_{K}^{12,3}(\rho, \vartheta)$ are orthonormal, when integrated over the angle - with a choice of integrating over a 'sector' (here the right-hand or left-hand part of the range) or over the whole range of 0 to $2 \pi$.

The $q_{K}$, here, are really $q_{K}^{12,3}$ and, of course, as in the fully interacting case, are also functions of $\rho$.

\section{$\Lambda$ 's, C's, D's}

Given the expressions for the $B$ 's ... we can then obtain expansions for the behaviour of the $q_{K}$ as functions of $\rho$, and therefore for $\Lambda_{K}$ and for the $C$ 's and the $D$ 's .... For us, the most important behaviour is that as $\rho$ becomes large .... i.e. expansions in $1 / \rho$.

We then find, [see Appendix B], that for $\rho \rightarrow \infty$ the effective centrifugal term (i.e. the $1 / \rho^{2}$ term) reads :

$$
\begin{aligned}
\Lambda_{K} & =\frac{(K+3)^{2}}{\rho^{2}}+\ldots \quad \text { for the case }(123) \\
\Lambda_{K}^{12,3} & =\frac{(K+1)^{2}}{\rho^{2}}+\ldots \quad \text { for the case }(12,3)
\end{aligned}
$$

and in both cases, the $C$ and $D$ terms behave as :

$$
\begin{gathered}
D_{K}^{K^{\prime}} \propto \begin{cases}\frac{1}{\rho^{3}}+\ldots & K \neq K^{\prime} \\
\frac{1}{\rho^{4}}+\ldots & K=K^{\prime}\end{cases} \\
C_{K}^{K^{\prime}} \frac{d}{d \rho} \propto \begin{cases}\frac{1}{\rho^{2}} \frac{d}{d \rho}+\ldots & K \neq K^{\prime} \\
0 & K=K^{\prime}\end{cases}
\end{gathered}
$$

In both cases, to obtain a non-zero result, the indices of the $K$ and $K^{\prime}$ matrix elements must both belong either to an order which is even or to an order which is odd. There are no cross terms.

\section{Coupled equations and asymptotic behaviour}

Given N coupled equations, we will have $\mathrm{N}$ independent solutions (well behaved at the origin) of the coupled differential equations. These can be expressed as:

$$
\tilde{\phi}^{K}(k, \vec{\rho})=\sum_{K^{\prime}} B_{K^{\prime}}(\rho, \vartheta) \phi_{K^{\prime}}^{K}(k, \rho)
$$

where the $\phi_{K^{\prime}}^{K}$ - the amplitudes - can be chosen to have as asymptotic behaviour, either:

$$
\phi_{K^{\prime}}^{K}(k, \rho)=\delta_{K^{\prime}}^{K} \sqrt{k \rho} J_{K^{\prime}+3}(k \rho)+W_{K^{\prime}}^{K}(k) \sqrt{k \rho} N_{K^{\prime}+3}(k \rho)
$$

or

$$
\bar{\phi}_{K^{\prime}}^{K}(k, \rho)=\delta_{K^{\prime}}^{K} \sqrt{k \rho} J_{K^{\prime}+1}(k \rho)+\bar{W}_{K^{\prime}}^{K}(k) \sqrt{k \rho} N_{K^{\prime}+1}(k \rho),
$$

as appropriate for the desired case.

[I.e. starting from amplitudes with $A_{K^{\prime}}^{K}$ and $B_{K^{\prime}}^{K}$ as coefficients of the Bessel and Neumann functions (multiplied by $\sqrt{k \rho}$ ), we take linear combinations of the solutions - multiplying by the matrix $A^{-1}$ - 
to obtain equations (19) and (20).] The upper index $K$, chosen to enumerate the solutions (18), is associated with the $J_{K}(k \rho)$ which dominates the solution for small values of $\rho$, and takes on the same set of values as the lower index $K^{\prime}$.

The wave functions and the amplitudes can be chosen to be real, and the $W_{K}^{K^{\prime}}$ and the $\bar{W}_{K}^{K^{\prime}}$ will be real and symmetrical. They can be diagonalized to yield tangents of eigenphase shifts $-\tan \delta_{W}^{K}(k)$ and $-\tan \left(\left(\bar{\delta}_{W}\right)^{K}\right)(k)$, respectively.

Further, we can then take linear combinations of these solutions, denoted as $\phi_{K^{\prime}}^{\nu}$, such that for the fully interacting system :

$$
\phi_{K^{\prime}}^{\nu}(k, \rho) \rightarrow(k \rho)^{1 / 2} \mathcal{C}_{K^{\prime}}^{\nu}(k)\left[\cos \delta_{W}^{\nu}(k) J_{K^{\prime}+3}(k \rho)-\sin \delta_{W}^{\nu}(k) N_{K^{\prime}+3}(k \rho)\right]
$$

where $K^{\prime}$ denotes the $K^{\prime}$ th amplitude of the solution $\nu$ and the eigenphase shifts are associated with the matrix $W$. In the fully interacting case, these phase shifts will only differ by $-3 \pi / 2$ from those of the genuine $S$-matrix for this problem. (I.e. $-3 \pi / 2+\delta_{W}=\delta_{S}$ )

In the $(12,3)$ case, we obtain similar expressions.

We note that since the ' $K$ ' $=$ even' amplitudes couple only with the ' $K^{\prime}=$ even' amplitudes and, analogously, the ' $K$ ' $=$ odd' amplitudes couple only with the ' $K$ ' $=$ odd' amplitudes, we can therefore separate our set of solutions $\phi_{K^{\prime}}^{\nu}$ into 2 sets, one with amplitudes involving only even values of $K^{\prime}$ and the other with amplitudes involving only odd values of $K^{\prime}$. 


\section{The phase shift expressions for the Traces}

\section{1st term - with the totally symmetric adiabatic (123) basis}

Consider the first term of the Eq.(41) :

$$
\frac{3^{1 / 2}}{\lambda_{T}}\left[\operatorname{Tr}\left(e^{-\beta H_{3}^{r e l}}\right)-\operatorname{Tr}\left(e^{-\beta T_{3}^{r e l}}\right)\right],
$$

seen here, after we have separated the Hamiltonian into two parts, one corresponding to the center of mass coordinate and the other to the relative motion, and taken the trace of the center of mass contribution. The latter contributes a factor that depends on the temperature $\lambda_{T}=\left(h^{2} / 2 \pi m \kappa T\right)^{1 / 2}$ and a length that cancels the factor $1 / L$ shown in Eq. (44). The first term of the above can now be written as

$$
\frac{3^{1 / 2}}{\lambda_{T}} \int d \vec{\rho} \int_{0}^{\infty} d k \sum_{\nu} \psi^{\nu *}(k, \vec{\rho}) \psi^{\nu}(k, \vec{\rho}) e^{-\beta\left(\hbar^{2} / 2 m\right) k^{2}}
$$

where we introduced a complete set of energy eigenfunctions, defined over the hyperspherical space $\vec{\rho}$.

As we have indicated before, we let $\psi^{\nu}=\tilde{\phi}^{\nu} / \rho^{1 / 2}$, and then expand the eigenfunction $\tilde{\phi}^{\nu}$ in terms of an adiabatic basis $B_{K}^{123}$, which is completely symmetric, complete and normalized over each of the intervals $(m \pi / 3-\pi / 6, m \pi / 3+\pi / 6)$, where $m=0,1, \cdots 5$. Each of these intervals is associated with one of the 6 possible permutations of the coordinates of the system. Integrating over just one of the sectors is to integrate over all of the possible configurations of the physical particles in a Bose system.

Alternatively, if we wish to integrate over the interval $-\pi$ to $\pi$ we can divide the angular functions by $\sqrt{3 !}$, taking into account their six-fold symmetry, and obtain a symmetric adiabatic base $B_{K}(\rho, \vartheta)$, normalized over this extended angular interval. Expanding the functions in terms of the symmetric adiabatic base, expression (23) now reads:

$$
\begin{gathered}
\frac{3^{1 / 2}}{\lambda_{T}} \int_{0}^{\infty} d k \int_{0}^{\infty} d \rho \sum_{\nu, K, K^{\prime}} \phi_{K}^{\nu *}(k, \rho) \phi_{K^{\prime}}^{\nu}(k, \rho) \\
\int_{-\pi}^{\pi} d \vartheta B_{K}^{*}(\rho, \vartheta) B_{K^{\prime}}(\rho, \vartheta) e^{-\beta\left(\hbar^{2} / 2 m\right) k^{2}}
\end{gathered}
$$

Here $\nu$ stands for the $\nu$ th solution and $K$, which takes on only even or only odd values for a given $\nu$, labels the components. Integration over $\vartheta$ then gives

$$
\frac{3^{1 / 2}}{\lambda_{T}} \int_{0}^{\infty} d k \sum_{\nu, K} \int_{0}^{\infty} d \rho \phi_{K}^{\nu *}(k, \rho) \phi_{K}^{\nu}(k, \rho) e^{-\beta\left(\hbar^{2} / 2 m\right) k^{2}}
$$

We note that had we expanded in terms of $B_{K}^{123}(\rho, \vartheta)$, and integrated over one of the sectors, we would have obtained the same expression.

The radial functions satisfy the coupled radial equations:

$$
\begin{aligned}
\left(\frac{d^{2}}{d \rho^{2}}-\Lambda_{K}(\rho)+k^{2}\right) \phi_{K}^{\nu}(k, \rho) & +2 \sum_{K^{\prime}} C_{K}^{K^{\prime}}(\rho) \frac{d}{d \rho} \phi_{K^{\prime}}^{\nu}(k, \rho) \\
& +\sum_{K^{\prime}} D_{K}^{K^{\prime}}(\rho) \phi_{K^{\prime}}^{\nu}(k, \rho)=0
\end{aligned}
$$

and we can choose our solutions to be real.

To evaluate the integral of the square of the wave function over $\rho$ we now use a procedure similar to that used in our previous papers 16 . We integrate the square of the wave functions within a sphere of radius $\rho_{\max }$, and then let $\rho_{\max }$ go to infinity. We use the Green's function 'trick' 3 , and obtain 


$$
\begin{gathered}
\int_{0}^{\rho_{\max }} \sum_{K}\left(\phi_{K}^{\nu}(k, \rho) \phi_{K}^{\nu}\left(k^{\prime}, \rho\right)\right) d \rho= \\
\frac{1}{k^{2}-\left(k^{\prime}\right)^{2}} \sum_{K}\left[\phi_{K}^{\nu}(k, \rho) \frac{d}{d \rho} \phi_{K}^{\nu}\left(k^{\prime}, \rho\right)-\phi_{K}^{\nu}\left(k^{\prime}, \rho\right) \frac{d}{d \rho} \phi_{K}^{\nu}(k, \rho)\right],
\end{gathered}
$$

evaluated at $\rho=\rho_{\max }$.

I.e. our identity is:

$$
\begin{aligned}
& \sum_{K} \frac{d}{d \rho}\left[\phi_{K}^{\nu}\left(k^{\prime}, \rho\right) \frac{d}{d \rho} \phi_{K}^{\nu}(k, \rho)-\phi_{K}^{\nu}(k, \rho) \frac{d}{d \rho} \phi_{K}^{\nu}\left(k^{\prime}, \rho\right)\right] \\
& +\left(k^{2}-\left(k^{\prime}\right)^{2}\right) \sum_{K} \phi_{K}^{\nu}(k, \rho) \phi_{K}^{\nu}\left(k^{\prime}, \rho\right) \\
& +2 \sum_{K, K^{\prime}} \frac{d}{d \rho}\left[\phi_{K}^{\nu}\left(k^{\prime}, \rho\right) C_{K}^{K^{\prime}}(\rho) \phi_{K^{\prime}}^{\nu}(k, \rho)\right]
\end{aligned}
$$

and we integrate with respect to $\rho$. Using then the fact that $\phi_{K}^{\nu}$ goes to zero, as $\rho$ itself goes to zero, and that $C_{K}^{K^{\prime}}$ decreases fast enough for $\rho$ large, we are left with the expression displayed earlier (that of our 'trick').

We now put in the real version of the asymptotic form of our solutions, Eq.(21), oscillatory solutions valid for $\rho_{\max }$ large, and use l'Hospital's rule to take the limit as $k^{\prime} \rightarrow k$. Thus for the interacting system

$$
\phi_{K}^{\nu}(k, \rho) \rightarrow(k \rho)^{1 / 2} \mathcal{C}_{K}^{\nu}(k)\left[\cos \delta_{W}^{\nu}(k) J_{K+3}(k \rho)-\sin \delta_{W}^{\nu}(k) N_{K+3}(k \rho)\right] .
$$

Here, we note again that we come to a major difference with what happens in other dimensions. We have seen, Eqs. (16), that for large values of $\rho$, the effective asymptotic 'centrifugal' term involves $K+3$, instead of the $K$ that appears for small values of $\rho$. The corresponding 'free' particle solution can be chosen to be:

$$
\left(\phi^{0}\right)_{K}^{\nu}(k, \rho)=(k \rho)^{1 / 2} \mathcal{C}_{K}^{\nu}(k) J_{K}(k \rho)
$$

We note that $\delta^{\nu}$ is associated with the solution $\nu$, and appears in each of the amplitudes of this particular solution. The coefficient $\mathcal{C}_{K}^{\nu}$, the mixture coefficient, tells us how much of each normalized amplitude appears in the solution. These two quantities therefore completely characterize the asymptotic behaviour of the $\nu$ th wave function. We expect that

$$
\sum_{K}\left(\mathcal{C}_{K}^{\nu}\right)^{*} \mathcal{C}_{K}^{\nu}=1
$$

(Or the real equivalent.)

Inserting this into our integrals, and taking the limit $k^{\prime} \rightarrow k$, we find that

$$
\sum_{K} \int_{0}^{\rho_{\max }}\left|\phi_{K}^{\nu}(k, \rho)\right|^{2} d \rho \rightarrow \frac{1}{\pi} \frac{d}{d k} \delta^{\nu}(k)+\frac{1}{\pi} \rho_{\max }+\text { osc.terms }
$$

and, thus, that

$$
\sum_{K} \int_{0}^{\rho_{\max }}\left(\left|\phi_{K}^{\nu}(k, \rho)\right|^{2}-\left|\left(\phi^{0}\right)_{K}^{\nu}(k, \rho)\right|^{2}\right) d \rho \rightarrow \frac{1}{\pi} \frac{d}{d k} \delta^{\nu}(k)+\text { osc.terms }
$$

We let $\rho_{\max }$ go to infinity, and usually the oscillating terms do not contribute to the integral over the energy. In 1 dimension, however, we have to be more careful. 
Using the asymptotic form of real amplitudes

$$
\sqrt{\frac{2}{\pi}} \mathcal{C}_{K}^{\nu}(k) \sin \left[k \rho-\frac{1}{2}(K+3) \pi+\frac{1}{4} \pi+\delta^{\nu}(k)\right]
$$

and l'Hospital's rule, when taking the limit as $k^{\prime} \rightarrow k$ in Eq.(27), we find that the integral over $\rho$, can be written as

$$
\frac{1}{\pi} \sum_{\nu}\left[\rho_{\max }+\frac{d}{d k} \delta^{\nu}(k)\right]+\frac{1}{2 \pi} \sum_{\nu, K}\left[\mathcal{C}_{K}^{\nu}(k)\right]^{2} \frac{1}{k} \sin \left[2\left(k \rho_{\max }-\frac{1}{2}(K+3) \pi+\frac{1}{4} \pi+\delta^{\nu}(k)\right)\right]
$$

When we subtract the term without interaction from the above expression, the integral over $\mathrm{k}$ takes the form, involving the eigenphases:

$$
\frac{3^{1 / 2}}{\lambda_{T}} \int_{0}^{\infty} d k e^{-\beta\left(\hbar^{2} / 2 m\right) k^{2}} \frac{1}{\pi} \sum_{\nu}\left[\frac{d}{d k} \delta^{\nu}(k)\right]
$$

and the contribution from the oscillating terms:

$$
\begin{aligned}
& \frac{3^{1 / 2}}{\lambda_{T}} \int_{0}^{\infty} d k \frac{1}{2 \pi} \sum_{\nu, K}\left(\mathcal{C}_{K}^{\nu}(k)\right)^{2}\left\{\frac { 1 } { k } \left(\sin \left[2\left(k \rho_{\max }-\frac{1}{2}(K+3) \pi+\frac{1}{4} \pi+\delta^{\nu}(k)\right)\right]\right.\right. \\
& \left.\left.-\sin \left[2\left(k \rho_{\max }-\frac{1}{2} K \pi+\frac{1}{4} \pi\right)\right]\right)\right\} e^{-\beta\left(\hbar^{2} / 2 m\right) k^{2}}
\end{aligned}
$$

which can be rewritten as

$$
\begin{gathered}
\frac{3^{1 / 2}}{\pi \lambda_{T}} \int_{0}^{\infty} d k \sum_{\nu}(\mp)_{\nu}\left\{\frac { 1 } { 2 k } \left(\cos \left[2\left(k \rho_{\max }\right)\right]\left[\cos \left(2 \delta^{\nu}(k)\right)+1\right]\right.\right. \\
\left.\left.-\sin \left[2\left(k \rho_{\max }\right)\right] \sin \left(2 \delta^{\nu}(k)\right)\right)\right\} e^{-\beta\left(\hbar^{2} / 2 m\right) k^{2}}
\end{gathered}
$$

where we have used the fact that the $K$ are either all even or all odd for a given eigenfunction $\nu$, giving a value of -1 to $\mp$, when the $\mathrm{K}$ are even and +1 when they are odd. For a given $\nu$, we can therefore sum over $K$. In fact, since we have isolated the $K$ dependence of the trigonometric terms, we can sum the $\left(\mathcal{C}_{K}^{\nu}\right)^{2}$, over $K$.

We should also mention that when we perform the subtraction of the 'free' wave function from the interacting one ... we must choose precisely the same $\mathcal{C}^{\prime} s$ for the free solution, as the ones that are mandated when we have an interaction! We are however free to do so!

The phase shifts, that appear in the expressions above, are the $W$-phase shifts ... that go to zero as $k$ tends to zero. In our last equation, therefore, the leading term of $\cos (2 \delta)+1$ will give +2 $\ldots$ and the individual integrands will 'blow' at the origin. We have a major problem! Unless our reasoning is incorrect, our only hope is that alternating signs and other similar oscillatory terms arising from the $(12,3)$ contributions - will enable us to precisely cancel what we have here. The term $\sin \left[2\left(k \rho_{\max }\right)\right] \sin \left(2 \delta^{\nu}(k)\right) / k$ will not contribute to the $k$-integral, when we take the limit $\rho_{\max } \rightarrow \infty$ 


\section{2nd term - with the symmetric adiabatic $(12,3)$ basis}

We take the second set of traces in Eq. (3) and rewrite it as an expression involving a trace of a 3-body operator:

$$
\operatorname{Tr}\left(e^{-\beta T_{1}}\right) \operatorname{Tr}\left(e^{-\beta H_{2}}\right)=\operatorname{Tr}\left(e^{-\beta\left(H_{2}+T_{1}\right)}\right)
$$

This is an essential trick. To obtain the L-independence of $b_{3}$, we need to subtract related quantities from all of the traces, i.e. evaluate them with same or similar harmonics or adiabatic functions, have sums which, though they might individually diverge if summed to infinity, can be subtracted to yield finite results. They can each be evaluated for a finite number of terms, subtracted by similar sums, and then permitted, for a fixed energy, to converge [This has worked for a Boltzmann calculation 8]] or - in our present case - subjected to an accelerated convergence procedure, subtracted and found to yield a finite result.

We now expand the wave functions, eigenstates of a 3-body Hamiltonian of 3 particles, for which, say, particles 1 and 2 are interacting, with particle 3 acting as a spectator, in terms of adiabatic angular functions and $\rho$-dependent amplitudes.

$$
\overline{\tilde{\phi}}^{\nu}(k, \vec{\rho})=\frac{1}{\rho^{1 / 2}} \sum_{K} B_{K}^{12,3}(\rho, \vartheta) \bar{\phi}_{K}^{\nu}(k, \rho)
$$

[We note that $\nu$ and $K$ are not the same indices as appear in the previous section, and do not assume the same sets of values.]

The angular functions, $B^{12,3}$, will be symmetric upon interchange of particles 1 and 2 , but will have no symmetry with respect to interchanges between particles 1 and 3 or 2 and 3 . They will be eigenfunctions of Eq.(6), with the potential $V$ equal to:

$$
\bar{V}=g \delta\left(\left|x_{1}-x_{2}\right|\right)=g \delta(\sqrt{2} \rho|\cos \vartheta|)
$$

and normalized over $1 / 2$ of the $\vartheta$ space, for example from $-\pi / 2$ to $+\pi / 2$, since this corresponds to a complete sampling of the possible physical configurations. Alternatively, we can define a $\overline{B_{l}}$ equal to $B^{12,3}$ divided by $\sqrt{2}$, which can be integrated from $-\pi$ to $+\pi$ and still be normalized. The equivalent to Eq.(24) is then

$$
\frac{3^{1 / 2}}{\lambda_{T}} \int_{0}^{\infty} d k e^{-\beta\left(\hbar^{2} / 2 m\right) k^{2}} \int_{0}^{\infty} d \rho \sum_{\nu, K, K^{\prime}}\left[\bar{\phi}_{K}^{\nu *}(k, \rho) \bar{\phi}_{K^{\prime}}^{\nu}(k, \rho) \int_{-\pi}^{\pi} d \vartheta \bar{B}_{K}^{*}(\rho, \vartheta) \bar{B}_{K^{\prime}}(\rho, \vartheta)\right]
$$

and the equivalent of Eq. (25) is then

$$
\frac{3^{1 / 2}}{\lambda_{T}} \int_{0}^{\infty} d k e^{-\beta\left(\hbar^{2} / 2 m\right) k^{2}} \sum_{\nu, K} \int_{0}^{\infty} d \rho \bar{\phi}_{K}^{\nu *}(k, \rho) \bar{\phi}_{K}^{\nu}(k, \rho)
$$

We can now subtract a trace with 'free' particles, with wave functions that are only symmetric under an interchange of particles 1 and 2, integrate over $\rho$ and define eigenphase shifts $\bar{\delta}^{\nu}(k)$ for this 3-body system, following steps similar to those of the previous section. There are differences, though. Instead of the $K+3$, which appeared in the asymptotic behaviour of $\Lambda_{K}$, we find that the effective asymptotic 'centrifugal' term involves $K+1$. The phase shifts must therefore be defined in terms of Bessel and Neumann functions of order $K+1$. They are, again, $W$-type phase shifts ... which go to zero as $k$ goes to zero.

For the contribution of the 2nd term, we then arrive at:

$$
\frac{3^{1 / 2}}{\lambda_{T}} \int_{0}^{\infty} d k e^{-\beta\left(\hbar^{2} / 2 m\right) k^{2}} \frac{1}{\pi} \sum_{\nu}\left[\frac{d}{d k} \bar{\delta}^{\nu}(k)\right]
$$


where the sum over $\nu$ must involve the even and odd values of $K$, but a particular $\bar{\psi}^{\nu}$ will only involve the even or the odd values of $K$. The oscillating terms yield:

$$
\begin{aligned}
& \frac{3^{1 / 2}}{\lambda_{T}} \int_{0}^{\infty} d k \frac{1}{2 \pi} \sum_{\nu, K}\left[\overline{\mathcal{C}}_{K}^{\nu}(k)\right]^{2}\left\{\frac { 1 } { k } \left(\sin \left[2\left(k \rho_{\max }-\frac{1}{2}(K+1) \pi+\frac{1}{4} \pi+\bar{\delta}^{\nu}(k)\right)\right]\right.\right. \\
& \left.\left.-\sin \left[2\left(k \rho_{\max }-\frac{1}{2} K \pi+\frac{1}{4} \pi\right)\right]\right)\right\} e^{-\beta\left(\hbar^{2} / 2 m\right) k^{2}}
\end{aligned}
$$

which can be rewritten as

$$
\begin{gathered}
\frac{3^{1 / 2}}{\pi \lambda_{T}} \int_{0}^{\infty} d k \sum_{\nu}(\mp)_{\nu}\left\{\frac { 1 } { 2 k } \left(\cos \left[2\left(k \rho_{\max }\right)\right]\left[\cos \left(2 \bar{\delta}^{\nu}(k)\right)+1\right]\right.\right. \\
\left.\left.-\sin \left[2\left(k \rho_{\max }\right)\right] \sin \left(2 \bar{\delta}^{\nu}(k)\right)\right)\right\} e^{-\beta\left(\hbar^{2} / 2 m\right) k^{2}}
\end{gathered}
$$

where, as before, $\mp$ takes the value of -1 when the $\mathrm{K}$ are even and +1 when they are odd.

We see that, just as in the previous oscillating terms for the 1 st term of $b_{3}$, again, the cosine term poses problems and the sine term does not contribute to the fugacity coefficient. 


\section{Distorted Born}

From the foregoing, we see that to obtain analytical results for $b_{3}-b_{3}^{0}$, we shall need an expansion of the eigenphase shifts, for small values of $k$, our customary wave number.

The 'conventional' result for a 2-body potential, as stated by Mott and Massey [17, is that given an orbital angular momentum $\ell \hbar$, and a radial potential that falls off as $r^{-s}$ for large $r$, then

$$
\lim _{k \rightarrow 0} k^{2 \ell+1} \cot \left(\eta_{\ell}\right)=\text { constant }
$$

if $\ell<(s-3) / 2$. This is the usual result, in three dimensions, for short range potentials. If, however, $\ell>(s-3) / 2$, Mott and Masey then obtain

$$
\lim _{k \rightarrow 0} k^{s-2} \cot \left(\eta_{\ell}\right)=\text { constant }
$$

a very different behaviour, that they show can be obtained by a Born approximation. The point is that, here, it is the long-tail behaviour of the potential that drives the low-energy behaviour of the phase shifts.

In our hyperspherical formalism we see, for repulsive delta-function interactions, from our equations (16), that when $\rho$ is large, the role of the $\ell+1 / 2$ is played by $K+3$ or $K+1$, respectively and, therefore, the criterion for the dominance of the 'tail' becomes $(K+3)>(s-2)$ for the $(123)$ case and $(K+1)>(s-2)$ when discussing the $(12,3)$ physical problem. We note that this is satisfied for our adiabatic potentials.

Still, the recommended use of a Born approximation is not so straightforward. This is due to a peculiarity arising from working in one dimension. In a manner not arising in other dimensions, for any of our equations, the effective centrifugal term changes as a function of $\rho$, due to the interaction. Thus, for the (123) case and a given $K$, for 'low' values of $\rho$ it assumes the centrifugal value of $\left(K^{2}-1 / 4\right) / \rho^{2}$, while for 'large' values of $\rho$, thanks to the interactions, it takes on the asymptotic value of $(K+3)^{2} / \rho^{2}$.

Thus a 'conventional' Born iteration, with the 'low- $\rho$ ' expression for the centrifugal term, and using $J_{K}(k \rho)$ as a zeroth order approximation for the solution, would yield iterates that would not form an expansion in powers of $k$, but would in fact each contribute to a constant term in a power series expansion of an R-matrix. [Here, to make the point, we just discuss one equation.]

We were then led to the development of the 'Distorted Born' [18, 13, 10].

For ample discussion, and details, see J.J. Popiel's thesis[13]. Below, we follow his general outline, for the case (123), which we have extended to include $K s$ that are odd, and also to the physics that we denote by $(12,3)$. We are happy to have recovered and extended his results; we simply note that we differ by a few signs (which, in some crucial equations, compensate!).

For low energies, we write:

$$
\left[\frac{d^{2}}{d \rho^{2}}-\frac{(K+3)^{2}-\frac{1}{4}}{\rho^{2}}+k^{2}\right] \phi_{K}^{K^{\prime}}(k, \rho)=\sum_{K^{\prime \prime}}(\quad)^{\infty} \phi_{K^{\prime \prime}}^{K^{\prime}}(k, \rho)
$$

where

$$
(\quad)_{K}^{K^{\prime \prime}}(\rho)=\left(\Lambda_{K}(\rho)-\frac{(K+3)^{2}}{\rho^{2}}\right) \delta_{K}^{K^{\prime \prime}}-D_{K}^{K^{\prime \prime}}(\rho)-2 C_{K}^{K^{\prime \prime}}(\rho) \frac{d}{d \rho} .
$$

The superscript $\infty$ on $(\quad)^{\infty}$ is meant to imply that we seek to use the large $\rho$ expansion of $(\quad)_{K}^{K^{\prime \prime}}$. Asymptotically, it will behave as $1 / \rho^{3}$. The matrix C, which only has off-diagonal elements, is proportional to $1 / \rho^{2}$, but only appears in the coupled equations as $C \frac{d}{d \rho}$ and hence for large $\rho$ is effectively proportional to $k / \rho^{2}$. The $K^{\prime}$ denotes the $K^{\prime}$ th solution.

An iteration of our coupled equations will yield a power series in $\mathrm{k}$ with each new term in the successive iteration beginning with a higher power of $\mathrm{k}$.

We now write an integral equation, valid for small values of $k$ :

$$
\phi_{K}^{K^{\prime}}(k, \rho)=\left(\phi_{K}^{K^{\prime}}\right)^{0}(k, \rho)+\int_{0}^{\infty} G_{K+3}\left(\rho, \rho^{\prime} ; k\right) \sum_{K^{\prime \prime}}(\quad)^{\infty} \phi_{K^{\prime \prime}}^{K^{\prime}}\left(k, \rho^{\prime}\right) d \rho^{\prime}
$$


where $\left(\phi_{K}^{K^{\prime}}\right)^{0}$ is the leading term in an expansion of $\phi_{K}^{K^{\prime}}$ for low energy. Our choice is

$$
\left(\phi_{K}^{K^{\prime}}\right)^{0}(k, \rho)=\delta_{K}^{K^{\prime}} \sqrt{k \rho} J_{K+3}(k \rho)
$$

and 1

$$
G_{K+3}\left(\rho, \rho^{\prime} ; k\right)=\left\{\begin{array}{ll}
+\frac{\pi}{2 k} \sqrt{k \rho} J_{K+3}(k \rho) \sqrt{k \rho^{\prime}} N_{K+3}\left(k \rho^{\prime}\right) & \rho<\rho^{\prime} \\
+\frac{\pi}{2 k} \sqrt{k \rho} N_{K+3}(k \rho) \sqrt{k \rho^{\prime}} J_{K+3}\left(k \rho^{\prime}\right) & \rho>\rho^{\prime}
\end{array} .\right.
$$

With this, the large $\rho$ behaviour of $\phi_{K}^{K^{\prime}}$ is

$$
\phi_{K}^{K^{\prime}}(k, \rho)=\delta_{K}^{K^{\prime}} \sqrt{k \rho} J_{K+3}(k \rho)+W_{K}^{K^{\prime}}(k) \sqrt{k \rho} N_{K+3}(k \rho)
$$

where ${ }^{1}$

$$
W_{K}^{K^{\prime}}(k)=+\frac{\pi}{2 k} \int_{0}^{\infty} \sqrt{k \rho} J_{K+3}\left(k \rho^{\prime}\right) \sum_{K^{\prime \prime}}()^{\infty} \phi_{K^{\prime \prime}}^{K^{\prime}}(k, \rho \prime) d \rho \prime
$$

We note that the matrix $\mathrm{W}$ now contains all of the scattering information beyond the $(k \rightarrow 0)$ result, and completely characterizes the asymptotic form of the wave-function..

Iterating once, gives us:

$$
W_{K}^{K^{\prime}}(k)=1 \text { st Distorted Born }+\ldots
$$

where $^{1}$

$$
\begin{aligned}
1 \text { st Distorted Born }= & +\left(\frac{\hbar^{2} k}{m g}\right)\left\{\frac{24 \sqrt{2}}{\pi} \frac{(K+3)\left(K^{\prime}+3\right)}{\left[\left(K+K^{\prime}+6\right)^{2}-1\right]\left[\left(K-K^{\prime}\right)^{2}-1\right]}\right\} \\
& +\delta_{K}^{K^{\prime}} \times\left(\frac{\hbar^{2} k}{m g}\right)^{2}\left\{\frac{\left(\frac{27}{\pi}+\frac{\pi}{12}\right)(K+3)}{(K+2)(K+4)}+\frac{9}{4 \pi(K+2)(K+3)(K+4)}\right\} \\
& +O\left(k^{3}\right)
\end{aligned}
$$

The 2nd Distorted Born yields a $k^{2}$ contribution which exactly cancels that obtained from the 1st Born! So, the total contribution of order $k^{2}$ is zero.

For the case $(12,3)$, and $\bar{W}_{K}^{K^{\prime}}$, the corresponding formula is:

$$
\begin{aligned}
1 \text { st Distorted Born }= & +\left(\frac{\hbar^{2} k}{m g}\right)\left\{\frac{8 \sqrt{2}}{\pi} \frac{(K+1)\left(K^{\prime}+1\right)}{\left[\left(K+K^{\prime}+2\right)^{2}-1\right]\left[\left(K-K^{\prime}\right)^{2}-1\right]}\right\} \\
& +O\left(k^{2}\right)
\end{aligned}
$$

The terms of $O\left(k^{2}\right)$ are very much more difficult to evaluate, as the individual diagonal terms for $K=0$ diverge! As we can see from Mott and Massey's discussion we are, for $K=0$ (and $s>3$, corresponding to higher inverse powers appearing also in the expansion of the interactions at large distances), at the limit of the domain of validity of this method of calculation.

In Appendix D we show for a simplified version of a model mentioned in K. Chadan and P.C. Sabatier's book [19], for which the exact solution is known, that our Distorted Born approach gives exactly the correct coefficients, where the Mott and Massey criterion states that it should (and which is also precisely where the integrals of the expansion converge).

\footnotetext{
${ }^{1}$ Change of sign from Popiel's result
} 


\section{The Phase Shift Sums}

from $W_{K}^{K}$

From Eqs. (33) and (40), we see that we need to evaluate

$$
\sum_{\nu}\left[\frac{d}{d k} \delta^{\nu}(k)\right]-\sum_{\nu^{\prime}}\left[\frac{d}{d k} \bar{\delta}^{\nu^{\prime}}(k)\right]
$$

To do so, we note that our phase shifts are those obtained from diagonalizations of our matrices of $W$ and $\bar{W}$, respectively. In each case, the sum of the tangents of their phase shifts forms a trace which is, of course, invariant under changes in the representation.

Thus we can, for example, write

$$
\sum_{\nu}\left[-\tan \left(\delta_{W}^{\nu}\right)\right]=\operatorname{Trace}(W)=\sum_{K} W_{K}^{K}
$$

but since we expand the phase shift in powers of $k$, and keep at most the first two terms, we find that we can evaluate Eq.(53) in first order Distorted Born, as:

$$
\frac{\hbar^{2}}{m g}\left(\frac{24 \sqrt{2}}{\pi}\right)\left[\sum_{0,3,6 \ldots} \frac{(K+3)^{2}}{4(K+3)^{2}-1}-\frac{1}{3} \sum_{0,1,2 \ldots} \frac{(K+1)^{2}}{4(K+1)^{2}-1}\right]
$$

where we have combined the cosine and the sine contributions.

As we can see, and as is appropriate for our clusters, the individual contributions from the (123) and the $(12,3)$ terms are infinite! We expect the difference to be finite.

Let us comment:

As shown in a semi-classical treatment [3, 17, each of the phase-shift sums corresponding to the above, in 3 dimensions, is associated with a classical expression integrand, which integrated over the position variables, diverges as the volume tends to infinity. When all of the terms of the cluster are taken together, the resulting integral is finite and volume independent.

\section{Abel summation}

We see, therefore, that the infinities are the result of taking the infinite volume limit ... and the differences of the phase shift sums must be taken with great care.

The literature of the evaluation and manipulation of divergent series is rich and of great intellectual interest. We give some references[20].

A famous method to evaluate such series is due to Abel. Using his method we introduce an additional parameter ' $x$ ', and powers of $x$, which will allow us to obtain finite results for each of our divergent sums (for $0<x<1$ ), subtract our sums, let $x=1$ and then revert to our 'physical' results.

We first start by rewriting the sums in (55) as

$$
\sum_{3,6 \ldots}^{\infty} \frac{K^{2}}{4 K^{2}-1}-\frac{1}{3} \sum_{1,2 \ldots}^{\infty} \frac{K^{2}}{4 K^{2}-1}
$$

Following Abel, we multiply the terms of the diverging sums by the strongly decreasing function $x^{K}$ where $0<x<1$

$$
\sum_{3,6 \ldots}^{\infty} x^{K} \frac{K^{2}}{4 K^{2}-1}-\frac{1}{3} \sum_{1,2 \ldots}^{\infty} x^{K} \frac{K^{2}}{4 K^{2}-1} .
$$


Here we see that for equal values of $K$, on both sides, say $K=3$, the same term appears in both sums, multiplied by $x$ to the same power. This is important.

Changing the index in the first sum, we obtain

$$
\sum_{1,2 \ldots}^{\infty} x^{3 K} \frac{K^{2}}{4 K^{2}-1 / 9}-\frac{1}{3} \sum_{1,2 \ldots}^{\infty} x^{K} \frac{K^{2}}{4 K^{2}-1}
$$

For the first term, then, since the series is absolutely convergent for $0<x<1$, we can write:

$$
\frac{1}{4} \sum_{0,3,6 \ldots}^{\infty} x^{K+3}+\frac{1}{16} \sum_{0,3,6 \ldots}^{\infty} x^{K+3}\left(\frac{1}{(K+3)^{2}-\frac{1}{4}}\right)
$$

Similarly, for the second sum, involving the $(12,3)$ terms, we write:

$$
\frac{1}{4} \sum_{0,1,2 \ldots}^{\infty} x^{K+1}+\frac{1}{16} \sum_{0,1,2 \ldots}^{\infty} x^{K+1}\left(\frac{1}{(K+1)^{2}-\frac{1}{4}}\right)
$$

The expression in the bracket in (55) then becomes:

$$
\frac{1}{4}\left(\sum_{0,3,6 \ldots}^{\infty} x^{K+3}-\frac{1}{3} \sum_{0,1,2 \ldots} x^{K+1}\right)+\frac{1}{16}\left(\sum_{0,3,6 \ldots}^{\infty} x^{K+3} \frac{1}{(K+3)^{2}-\frac{1}{4}}-\frac{1}{3} \sum_{0,1,2 \ldots}^{\infty} x^{K+1} \frac{1}{(K+1)^{2}-\frac{1}{4}}\right)
$$

Collecting the divergent parts, gives us

$$
\sum_{0,3,6 \ldots}^{\infty} x^{K+3}-\frac{1}{3} \sum_{0,1,2 \ldots} x^{K+1}=\frac{x^{3}}{1-x^{3}}-\frac{1}{3} \frac{x}{1-x}=-\frac{x+2 x^{2}}{3\left(1+x+x^{2}\right)}
$$

which tends to $-1 / 3$ when $x \rightarrow 1^{-}$.

On the other hand

$$
\sum_{0,3,6 \ldots}^{\infty} \frac{1}{(K+3)^{2}-\frac{1}{4}}=\frac{1}{9} \sum_{1,2,3 \ldots}^{\infty} \frac{1}{j^{2}-\frac{1}{36}}=2-\frac{\pi}{\sqrt{(3)}}
$$

and

$$
\sum_{0,1,2 \ldots}^{\infty} \frac{1}{(K+1)^{2}-\frac{1}{4}}=\sum_{1,2,3 \ldots}^{\infty} \frac{1}{j^{2}-\frac{1}{4}}=2
$$

So that we obtain for (55)

$$
\frac{\hbar^{2}}{m g} \frac{24 \sqrt{(2)}}{\pi}\left\{-\frac{1}{4} \times \frac{1}{3}+\frac{1}{16}\left(2-\frac{\pi}{\sqrt{(3)}}-\frac{1}{3} 2\right)\right\}=-\frac{\hbar^{2}}{m g} \frac{\sqrt{6}}{2}
$$




\section{The oscillatory terms}

\section{Abel summation applied to the sums for the oscillating terms.}

Let us use the Abel procedure for the elements of the sums that appear in the integrands of the oscillating terms. I.e., recall that in (35) and (42) we obtained a term such as $(\mp)^{\nu}\left[\cos \left(2 \delta^{\nu}(k)\right)+1\right]$, which gives a -2 for the even $K$ terms and a 2 for the odd $K$ terms, as $k \rightarrow 0$. Using, then a 'trace' argument, we pass from the sums over $\nu$ to sums over the indices $K$ associated with our solutions. I.e., if we take one of the $W$ - associated with one of the sums in the oscillatory terms - say for a (123), even basis - and diagonalize it, we will then obtain $\delta^{K}$ for a set of values of $K$, such as $0,6,12, \ldots$, etc. We can use these values of $K$ to be our values of $\nu$, i.e. to be the values of the index over which we sum.

We will then have

$$
\sum_{0,6,12 \ldots}^{\infty} x^{K+3}-\sum_{3,9,15 \ldots}^{\infty} x^{K+3}-\sum_{0,2,4 \ldots} x^{K+1}+\sum_{1,3,5 \ldots} x^{K+1}
$$

which gives

$$
\frac{x^{3}\left(1-x^{3}\right)}{1-x^{6}}-\frac{x(1-x)}{1-x^{2}}=\frac{x^{3}}{1+x^{3}}-\frac{x}{1+x}
$$

then

$$
\frac{x^{3}}{1+x^{3}}-\frac{x\left(1-x+x^{2}\right)}{1+x^{3}}=\frac{x(x-1)}{1+x^{3}}
$$

which goes to 0 as $x \rightarrow 1$.

Further, if in the integral one makes the change of variable $u=k \rho_{\max }$, then when one takes the limit $\rho_{\max } \rightarrow \infty$, the argument of the $\delta^{\nu}\left(u / \rho_{\max }\right)$ will go to zero, and if we take the appropriate sums we find ourselves with the same expressions that we have evaluated above. The integrand of the total oscillatory contribution will be exactly zero. 


\section{The Fugacity Coefficient}

\section{our result}

Our fundamental formula is:

$$
b_{3}-b_{3}^{0}=\frac{3^{1 / 2}}{\lambda_{T}} \int_{0}^{\infty} d k e^{-\beta\left(\hbar^{2} / 2 m\right) k^{2}} \frac{1}{\pi}\left(\sum_{\nu} \frac{d}{d k} \delta^{\nu}(k)-\sum_{\nu \prime} \frac{d}{d k} \bar{\delta}^{\nu \prime}(k)\right)
$$

Putting the values that we have just obtained (60) for the derivative of the difference of the phase shift sums, we obtain:

$$
\frac{3^{1 / 2}}{\lambda_{T}}\left(-\frac{1}{\pi} \frac{\hbar^{2}}{m g} \frac{\sqrt{6}}{2}\right) \int_{0}^{\infty} d k e^{-\beta\left(\frac{\hbar^{2}}{2 m}\right) k^{2}}
$$

and remembering that $\lambda_{T}$ can also be written as $\left(2 \pi \hbar^{2} \beta / m\right)^{1 / 2}$ we obtain our result:

$$
b_{3}-b_{3}^{0}=-\frac{3 \sqrt{2}}{4 \pi g} \frac{1}{\beta},
$$

which agrees with the first term of the expansion of the integrals of Dodd and Gibbs 4 .

See Appendix C. 


\section{Conclusion}

Our aim, our hope, is to evaluate, express the virial coefficients in terms of scattering, asymptotic quantities of the wave functions, and bound state energies.

This was done in the 1930's for the 2nd virial by Uhlenbeck and Beth 21, and by Gropper 22. Our dream has been to do the same thing for the 'higher' virials. We believe that we have made progress in doing so for all the 'higher' virials - since our approach can be generalized to accommodate any number of particles - but demonstrate our method for a prototype third fugacity cluster, required for the 3rd virial.

In this paper, we present results for a model in 1-dim. (with delta function interactions) because this model has attracted the attention of many workers [23, 24, 25], due to its simplicity, which allows the evaluation of many results, expressions, and steps, analytically. Further, Dodd and Gibbs 4, uniquely, were able to look at the Statistical Mechanics and evaluate the third fugacity cluster in 1-dim. and we anticipated that we would be able to compare with their result.

In essence, our overall reasoning is simple and, we think, transparent!

Our fugacity clusters are expressed in terms of traces of terms such as $\operatorname{Tr}\left(e^{-\beta H_{n}}\right)$. We know that we can introduce complete sets of position eigenstates in these traces, which will then require us to integrate over position variables, and a wave number coordinate, as done for our model by Dodd and Gibbs 4 .

We do introduce complete sets of states, which we choose to expand in a hyperspherical adiabatic basis, and then integrate analytically over all position coordinates - including our hyper-radius coordinate $\rho$ - obtaining results characterized, here, by the asymptotic behaviour of the wave functions (i.e. for large $\rho$ ), described by eigenphase shifts, functions of the wave number.

This approach is general. Yes, there are subtle problems involving bound states, but here, in our 1-dim. repulsive case, we need not face them. In 1-dim., however, we find that there is a possibility of a contribution from oscillating terms, which for example do contribute[16] to $b_{2}$, in the case of Bose statistics. Our results indicate that there is no such contribution for $b_{3}$ in Bose statistics.

A delicate problem that we face, here, is the subtraction between phase shift sums. As we have shown, after evaluating the c.m. contribution, we must subtract contributions proportional to $L^{2}$, so as to obtain $L$-independent results. In our formalism, the first subtraction takes place, eliminating one power of $L$ and yielding 2 types of $\mathrm{W}$-phase shifts. We then saw that we had to subtract these two phase shifts sums to yield a finite, i.e. L-independent, answer. Using the results only of order $k$, we were successful in doing so, using a summation procedure associated with Abel.

For our 3 particles in one dimension, subject to delta-function interactions, we were able, for the fully interacting case (123), using a distorted Born approximation, to obtain the phase shifts (or their tangents), to order $k^{2}$. In our hyperspherical adiabatic formalism, they are associated with the 'tail' of long ranged potentials.

For the $(12,3)$ case, we encountered reliable results for the lowest contribution, to order $k$. However, for $K=0$, and the coefficient of $k^{2}$, we obtain divergences from the contributing terms. Added together, these divergences cancel but yield a finite term that we cannot trust. See, in Appendix D, our analysis of the domain of applicability of the distorted Born. [We note that we tried - very hard, in many ways, using different methods - to obtain the coefficient of the $k^{2}$ term, but could not.]

Using, then, the result of order $k$, which we do believe is correct in our distorted Born formalism, we obtain a result, for the temperature behaviour of $b_{3}$, which agrees with the leading term in an expansion of the double integral proposed by Dodd and Gibbs.

We feel justified, therefore, in believing in the validity of our formalism, and of its extension to 1-dim. and Bose particles.

Ultimately, in our future plans, we will wish to extend, rigorously, our general formalism, to include cases of three-body systems with attractive potentials and bound states. Specifically, we need to be able to accommodate states in which a possible outcome, asymptotically, would involve, for example, 
a 2-body bound state + an asymptotically free particle. In an old paper 3 , we proposed a formula to do this, a very intuitive formula, without a proper proof. Surely, one of our aims must to be remedy that!

(We note that to take into account a 3-body bound state is trivial; in the evaluation of the trace we simply add an exponential term involving the bound state energy.) 


\section{Acknowledgments}

A. Amaya and S. Y. Larsen acknowledge Dirección General de Asuntos del Personal Académico for partial support under project No. IN-105707-3. S. Y. Larsen also gratefully thanks the Instituto de Ciencias Físicas and the Institut de Physique Nucléaire for their always splendid hospitality. 


\section{Appendix A}

See the discussion in the article by Larsen and Poll [26].

$$
\phi_{j}^{i}(k, \rho) \sim(k \rho)^{1 / 2}\left(\delta_{j}^{i} J_{j}(k \rho)+R_{j}^{i}(k) N_{j}(k \rho)\right)
$$

As the reactance matrix $\left\|R_{j}^{i}\right\|$ is real and symmetric, it can be diagonalized by a real orthogonal matrix $\left\|\mathcal{C}_{j}^{i}\right\|$. Consequently, we can introduce eigensolutions:

$$
\phi_{j}^{\nu}(k, \rho) \sim(k \rho)^{1 / 2} \mathcal{C}_{j}^{\nu}\left(J_{j}(k \rho)+\Lambda_{\nu}(k) N_{j}(k \rho)\right)
$$

characterized by a unique eigenvalue for all of its amplitudes.

The eigenphase shifts are then defined by:

$$
\Lambda_{\nu}(k)=-\tan \delta_{\nu}(k)
$$




\section{Appendix B}

In this Appendix we give the asymptotic expression of the interaction ()$_{K}^{K^{\prime}}$ entering the coupled equations (Eqs. (43/44)). It consists of a series in terms of $1 / \rho$ and is used in our first and second Born approximations. We put for the (123) case

$$
q_{K}=(K+3)+\sum_{j} \frac{a_{j}}{\rho^{j}}
$$

with $K=0$ modulo 6 for $K$ even, and $K=3$ modulo 6 for $K$ odd, and for the $2+1$ case

$$
q_{K}=(K+1)+\sum_{j} \frac{\bar{a}_{j}}{\rho^{j}}
$$

with $K=0$ modulo 2 for $K$ even, and $K=1$ modulo 2 for $K$ odd. This leads to (see the previous discussions on the calculations of the adiabatic eigenfunctions)

$$
q_{K}=(K+3)-36\left(\frac{K+3}{c \pi^{2} \rho}\right)+1296\left(\frac{K+3}{c^{2} \pi^{4} \rho^{2}}\right)+\mathcal{O}\left(\frac{1}{\rho^{3}}\right)
$$

for every value of $K$ in the (123) case and

$$
q_{K}=(K+1)-12\left(\frac{K+1}{c \pi^{2} \rho}\right)+144\left(\frac{K+1}{c^{2} \pi^{4} \rho^{2}}\right)+\mathcal{O}\left(\frac{1}{\rho^{3}}\right)
$$

for every value of $K$ in the $(12,3)$ case.

In both cases $c=\left(2 m / \hbar^{2}\right)(3 g / \pi \sqrt{2})$.

Obtaining the asymptotic expansion of $\Lambda_{K}, C$ and $D$ matrices (See definitions in Eqs. (6/9]10)) in terms of $1 / \rho$, we recover (and extend) the results for (123) of Larsen and Popiel [10].:

$$
\begin{gathered}
\Lambda_{K}(\rho)=\left(q_{k}^{2}-1 / 4\right) / \rho^{2}=\frac{(K+3)^{2}-1 / 4}{\rho^{2}}+\mathcal{O}\left(\frac{1}{\rho^{3}}\right) \\
C_{K}^{K^{\prime}}(\rho)=-\frac{d}{\rho^{2}} \frac{12 \sqrt{2}(K+3)\left(K^{\prime}+3\right) \cos \left(\pi\left(K-K^{\prime}\right) / 6\right)}{\left(K-K^{\prime}\right)\left(K+K^{\prime}+6\right) \pi} \\
+\frac{d^{2}}{\rho^{3}} \frac{144(K+3)\left(K^{\prime}+3\right) \cos \left(\pi\left(K-K^{\prime}\right) / 6\right.}{\left(K-K^{\prime}\right)\left(K+K^{\prime}+6\right) \pi^{2}}+\mathcal{O}\left(\frac{1}{\rho^{4}}\right)
\end{gathered}
$$

and

$$
\begin{aligned}
D_{K}^{K^{\prime}}(\rho) & =\frac{d}{\rho^{3}} \frac{24 \sqrt{2}(K+3)\left(K^{\prime}+3\right) \cos \left(\pi\left(K-K^{\prime}\right) / 6\right)}{\left(K-K^{\prime}\right)\left(K+K^{\prime}+6\right) \pi} \\
& -\frac{d^{2}}{\rho^{4}} \frac{144(K+3)\left(K^{\prime}+3\right)\left(5(K+3)^{2}-\left(K^{\prime}+3\right)^{2}\right) \cos \left(\pi\left(K-K^{\prime}\right) / 6\right)}{\left(K-K^{\prime}\right)^{2}\left(K+K^{\prime}+6\right)^{2} \pi^{2}}+\mathcal{O}\left(\frac{1}{\rho^{5}}\right)
\end{aligned}
$$

and for $K=K^{\prime}$

$$
D_{K}^{K}(\rho)=-\frac{d^{2}}{\rho^{4}} \frac{2\left(27+(K+3)^{2} \pi^{2}\right)}{3 \pi^{2}}+\mathcal{O}\left(\frac{1}{\rho^{5}}\right)
$$

[Note that $C_{K}^{K^{\prime}}$ is an antisymmetric matrix and that, therefore, the diagonal elements are zero.]

In these expressions $d=\hbar^{2} / m g$. 
We obtain in the new case, $(12,3)$,

$$
\begin{gathered}
\Lambda_{K}(\rho)=\frac{(K+1)^{2}-1 / 4}{\rho^{2}}+\mathcal{O}\left(\frac{1}{\rho^{3}}\right) \\
C_{K}^{K^{\prime}}(\rho)=-\frac{d}{\rho^{2}} \frac{4 \sqrt{2}(K+1)\left(K^{\prime}+1\right) \cos \left(\pi\left(K-K^{\prime}\right) / 2\right)}{\left(K-K^{\prime}\right)\left(K+K^{\prime}+2\right) \pi} \\
+\frac{d^{2}}{\rho^{3}} \frac{16(K+1)\left(K^{\prime}+1\right) \cos \left(\pi\left(K-K^{\prime}\right) / 2\right)}{\left(K-K^{\prime}\right)\left(K+K^{\prime}+2\right) \pi^{2}}+\mathcal{O}\left(\frac{1}{\rho^{4}}\right)
\end{gathered}
$$

and

$$
\begin{aligned}
D_{K}^{K^{\prime}}(\rho) & =\frac{d}{\rho^{3}} \frac{8 \sqrt{2}(K+1)\left(K^{\prime}+1\right) \cos \left(\pi\left(K-K^{\prime}\right) / 2\right)}{\left(K-K^{\prime}\right)\left(K+K^{\prime}+2\right) \pi} \\
& -\frac{d^{2}}{\rho^{4}} \frac{16(K+1)\left(K^{\prime}+1\right)\left(5(K+1)^{2}-\left(K^{\prime}+1\right)^{2}\right) \cos \left(\pi\left(K-K^{\prime}\right) / 2\right)}{\left(K-K^{\prime}\right)^{2}\left(K+K^{\prime}+2\right)^{2} \pi^{2}}+\mathcal{O}\left(\frac{1}{\rho^{5}}\right)
\end{aligned}
$$

for $K \neq K^{\prime}$, and for $K=K^{\prime}$ we have

$$
D_{K}^{K}(\rho)=-\frac{d^{2}}{\rho^{4}} \frac{2\left(3+(K+1)^{2} \pi^{2}\right)}{3 \pi^{2}}+\mathcal{O}\left(\frac{1}{\rho^{5}}\right)
$$




\section{Appendix C}

\section{Expansion of the integrals of Dodd and Gibbs}

For their concluding equation Eq.(18), in the main part of their paper, Dodd and Gibbs [4] write:

$$
b_{3}-b_{3}^{0}=\frac{6}{(2 \pi)^{3}}\left(\frac{\pi}{\beta}\right)^{3 / 2} \int_{0}^{\infty} d s \int_{0}^{\infty} d t e^{-(1 / 2 \beta)\left(s^{2}+t^{2}+s t\right)}\left(e^{-c s-c t}-1\right) e^{-c s}
$$

Denoting, the integral above as $\mathcal{I}$, the integral can be rewritten as

$$
2 \beta \int_{0}^{\infty} d s \int_{0}^{\infty} d t \exp \left(-\left(s^{2}+t^{2}+s t\right)\right)(\exp (-\lambda(s+t))-1) \exp (-\lambda s),
$$

where we have set $\lambda=c \sqrt{2 \beta}$, and written again as:

$$
\text { (2ß) } \frac{\sqrt{\pi}}{2} \int_{0}^{\infty} d s e^{-s^{2}}\left\{e^{-2 \lambda s+\frac{(\lambda+s)^{2}}{4}} \operatorname{erfc}\left(\frac{\lambda+s}{2}\right)-e^{-\lambda s+\frac{s^{2}}{4}} \operatorname{erfc}\left(\frac{s}{2}\right)\right\}
$$

This then leads to the following expansion:

$$
\mathcal{I} /(2 \beta) \simeq-\frac{\sqrt{\pi}}{2 \lambda}+\frac{1}{\lambda^{2}}+\frac{3 \sqrt{\pi}}{4 \lambda^{3}}-\frac{4}{\lambda^{4}}+\mathcal{O}\left(\frac{1}{\lambda^{5}}\right)
$$

Taking the first term, we obtain:

$$
b_{3}-b_{3}^{0}=\frac{6}{(2 \pi)^{3}}\left(\frac{\pi}{\beta}\right)^{3 / 2}(2 \beta)\left(-\frac{\sqrt{\pi}}{2 c \sqrt{2 \beta}}\right)=-\frac{3}{4 \pi \sqrt{2}} \frac{1}{\beta c}
$$

Looking then at the two papers, we determine that $\beta$ of DG equals the $\left(\hbar^{2} / 2 m\right) \beta$ that we use, and that the $2 c$, used above, is our $g\left(2 m / \hbar^{2}\right)$. Substituting then, in their result, gives our formula:

$$
b_{3}-b_{3}^{0}=-\frac{3}{4 \pi \sqrt{2}} \frac{1}{g \beta} .
$$




\section{Appendix D}

\section{A little model, testing Distorted Born}

Consider the following differential equation:

$$
\left(-\frac{d^{2}}{d r^{2}}+\frac{2 a^{2}}{(1+a r)^{2}}\right) \psi(k, r)=k^{2} \psi(k, r) .
$$

I.e., a Schrödinger equation with a curious potential which, while finite at the origin, tends to $2 / r^{2}$ at large distances. Thus, it mimics the situation that we have encountered in this work, in which the 'effective' centrifugal term has changed as a function of distance.

The exact solution is:

$$
\psi(k, r)=\frac{-a^{2}(k r) \cos (k r)+\left(a^{2}+k^{2}+a k^{2} r\right) \sin (k r)}{k^{2}(1+a r)}
$$

which, for $r$ large, becomes proportional to

$$
\sqrt{k r}\left[\frac{a}{k} J_{3 / 2}(k r)-N_{3 / 2}(k r)\right]
$$

which implies that, introducing a $W$ and a phase shift $\delta$, we obtain an exact result: $W=-k / a$, and therefore $\tan (\delta)=k / a$. There are no terms in $k^{2}$, or $k^{3}$ for the tangent of $\delta$.

For $r$ large, the potential takes the form $2 / r^{2}-(4 / a) 1 / r^{3}+\left(6 / a^{2}\right) 1 / r^{4} \ldots$ and using the $-(4 / a) 1 / r^{3}$ in our first distorted Born yields

$$
\frac{\pi}{2 k} \int_{0}^{\infty} d r \sqrt{k r} J_{3 / 2}(k r) \sqrt{k r} J_{3 / 2}(k r) \frac{(-4 / a)}{r^{3}},
$$

which equals

$$
-\frac{2 \pi}{a} k \int_{0}^{\infty} d z\left(J_{3 / 2}(z)\right)^{2} \frac{1}{z^{2}}=-\frac{k}{a} .
$$

We note that the integrand behaves as $z$ at the origin - hence presents no difficulty there - and so would a term in the potential of $1 / r^{4}$, which would give $1 / z^{3}$ and an integrand which would be finite at the origin. A term in the potential of $1 / r^{5}$ would lead to a divergence.

If we use our distorted Born formulation to 2nd order, we obtain :

$$
W=-k / a+(k / a)^{2}(2 \pi / 5-2 \pi / 5)=-k / a
$$

where the 1 st $2 \pi / 5$ comes from the $1 / r^{4}$ term in the 1 st Born, and the 2 nd $2 \pi / 5$ from the 2 nd Born.

We see that in this little model, when the integrals converge, we obtain the correct answer to order $k^{2}$. It is the $k^{3}$ term in this model (for $\mathrm{W}$ ) which plays the role of the $k^{2}$ term in our $(2+1)$ case. I.e. in these cases [model and $(2+1)$ ] the relevant integrals diverge.

We note that the criterion, given by Mott and Massey for the dominance of the tail in determining the low $k$ behaviour, is that $\ell>(s-3) / 2$, where $s$ characterizes the inverse power of $\mathrm{r}$ being considered. Here, our divergent term is associated with $\ell=1$ and $s=5$. The inequality is thus violated and the Born (and also our distorted Born) has reached the limit of its application.. 


\section{References}

[1] T. Kinoshita, T. Wenger and D. S. Weiss. Nature, 440/13, 900 (2006).

[2] N. A. R. Hussien and A. A. Yahya. J. Phys. A: Math. Gen., 30, 445 (1997).

[3] S. Larsen. arXiv:physics/0105074v1; Paper presented at the Bogoliubov Conference on Problems of Theoretical and Mathematical Physics, 1999. Phys. Elem. Part. and Atom. Nucl., Part. and Nucl., 31 7b, 156 (2000).

[4] L. R. Dodd and A. M. Gibbs. J. Math. Phys., 15, 41 (1974).

[5] D. Dashen, S. Ma and M.J. Bernstein. Phys. Rev., 187, 345 (1969).

[6] S.Y. Larsen and P.L. Mascheroni. Phys. Rev. A, 2, 1018 (1970).

[7] S.Y. Larsen, A. Palma and M. Berrondo. J. Chem. Phys., 77, 5816 (1982).

[8] S.Y. Larsen and J. Zhen. Mol. Phys., 63, 581 (1988).

[9] W. Gibson, S.Y. Larsen, and J. Popiel. Phys Rev. A, 35, 4919 (1987).

[10] J.J. Popiel and S.Y. Larsen. Few-Body Systems, 15, 129 (1993).

[11] A. Amaya-Tapia, S.Y. Larsen, and J. Popiel. Few-Body Systems, 23, 87 (1997).

[12] S. Larsen. The Hyperspherical way. Few-Body Methods, ed. TK Lim, pp. 467-506. Singapore: World Scientific 1986.

[13] J.J. Popiel. Thesis Temple University 1991.

[14] Chuluunbaatar, A. A. Gusev, S. Y. Larsen, and S. I. Vinitsky. J. Phys. A : Math. and Gen. 35 pp. L513-L525 (2002).

[15] O. Chuluunbaatar, A. A. Gusev, M. S. Kaschiev, V. A. Kaschieva, A. Amaya-Tapia, S. Y. Larsen and S. I. Vinitsky. J. Phys. B: At. Mol. Opt. Phys.,39 pp. 243-269 (2006); A. A. Gusev,O. Chuluunbaatar, D.V. Pavlov, S. Y. Larsen and S. I. Vinitsky. J. Comp. Methods Sci. Eng. 2 pp. 1-3 (2003); S. I. Vinitsky, S. Y. Larsen, D.V. Pavlov and D.V. Proskurin Phys. At. Nuc. 64 pp. 27-37 (2001).

[16] A. Amaya-Tapia, S.Y. Larsen, and M. Lassaut. Mol. Phys., 103, pp.1301-1306 (2005).

[17] N.F. Mott and H.S.W. Massey. The theory of Atomic Collisions, 3rd Ed., pp43,89. Oxford University Press 1965.

[18] S.Y. Larsen and J.J. Popiel. Proc. of the 12th Int. Conf. on Few-Body Problems in Physics, B.K. Jennings Editor, p.15 Vancouver, TRIUMPH 1989.

[19] K. Chadan and P.C. Sabatier. Inverse Problems in Scattering Theory, 2nd Ed. (Springer, Berlin, 1989) .

[20] E. Borel. Leçons sur les séries divergentes, 2nd Ed. (Gauthiers-Villars, Paris, 1928); G.H. Hardy. Divergent Series, Oxford at the Clarendon Press, 1949; M. Zamansky. La sommation des séries divergentes, (Gauthiers-Villar, Paris, 1954); W. B. Ford. Studies on divergent series and summability, (Chelsea, New York, 1960).

[21] G. E. Uhlenbeck and E. Beth, Physica 3, 729 (1936); E. Beth and G. E. Uhlenbeck, ibid. 4, 915 (1937).

[22] L. Gropper, Phys. Rev. 50, 963 (1936); 51, 1108 (1937). 
[23] J. B. McGuire, J. Math. Phys. 5, 622 (1964); J. Math. Phys. 6, 432 (1965); J. Math. Phys. 7, 507 (1972).

[24] C. N. Yang, Phys. Rev. 168, 1920 (1968); C. N. Yang, C. P. Yang, J. Math. Phys. 10, 1115 (1969); C. P. Yang, Phys. Rev. A2, 154 (1970).

[25] L. R. Dodd, J. Math. Phys. 11, 207 (1970); Aust. J. Phys. 25, 607 (1972).

[26] S.Y. Larsen and J.D. Poll. Can. J. Phys., 52, 1914 (1974). 